\title{
Physiotherapy after ankle distorsion from inversion type
}

\author{
Victor Valchanov ${ }^{1}$, Stefan Yanev², \\ Svetoslav Garov ${ }^{3}$
}

1. FC "Tsarsko selo";

2. Department of Kinesitherapy, Faculty of Public Health, Medical University - Sofia;

3. Department of Medical Pedagogy, Faculty of Public Health, Medical University - Sofia;

\begin{abstract}
Footballers in their sports career receive a number of injuries, some of which are repetitive. The most burdened joints - the knee and the ankle and they are at the same time most traumatized. In turn, one of the most common pathologies of the talocrural joint is the distortion and rupture of the anterior talofibular and the calcaneofibular ligament. The trauma usually occurs when trying to steal the ball from an opposing player. The mechanism is by transferring the weight of the body onto one leg with simultaneous inversion of the foot.
\end{abstract}

Keywords: physiotherapy, distorsio, PNF, anterior talofibular ligament, calcaneofibular ligament 


\section{Introduction}

The ankle joint is a complex joint. It is composed of a talcrual, subtalar and talocalcaneonavicular joint. In the first one, movements are made mainly (because of its conical shape and the oblique axis) in the sagittal plane, in the second and the third in the transverse and the frontal plane. Several groups of ligaments and tendons are used to stabilize these joints. The main ligament between the talus and the calcaneus is lig. talocalcaneum interosseum. The lateral stabilization is performed by lig. talocalcaneumlaterale. From the front side lig. talocalcaneumanterior. The medial joint is stabilized by pars tibiocalcanea lig. deltoidea. The talocrural joint itself haslateral stabilization by lig. tibiofibulare anterius, lig. tibiofibulare posterius, lig. talofibulare anterius and lig. calcaneofibulare, andform medial side by lig. deltoideum.

The active stabilization of the ankle joint is performed by four groups of muscles:

- Front group: m.tibialis anterior, m.extensor digitorum longus, m.extensor hallucis longus and m.peroneus tertius.

- Lateral group: m.peroneus longus and m.peroneus brevis.

- Rear surface group: m.gastrocnemius, m.soleus and m.plantaris.

- Rear deep group: m.tibialis posterior, m.flexor digitorum longus, and m.flexor hallucis longus

\section{Pathobiomechanics of ligaments injury}

One of the external factors contributing to the damage to the lateral ligament stabilizing complex is sports injuries. It is also conditioned by several internal factors - the different form of trochlea tali, anatomical variations in lig. tibiofibulare anterius and lig. calcaneofibulare.

From the biomechanical point of view, the most stable position of the ankle joint is maximal dorsal flexion and pronation, and the most unstable - maximal plantar flexion with supination. Precisely, the second type it is most common injuries of the lower limb, caused in $90 \%$ of cases by the so-called "Varus/inversion" trauma (stepping/falling of the lateral end of the foot). Depending on the position of the ankle during trauma, the following ligaments are most often damaged:

- anterior talofibular ligament - plantar flexion with inversion;

- calcaneofibular ligament - dorsal flexion with inversion;

- posterior talofibular ligament;

- calcaneocuboid ligament.

Trauma of the ankle usually results in a combination of injuries to the above mentioned ligaments, as well as tibiofibular syndesmosis, and the front capsule. We often have associated lesions of both the sensory nerves (n.suralis and the superficial fibular nerve involved in the capsule innervation) or the fibular tendons. 


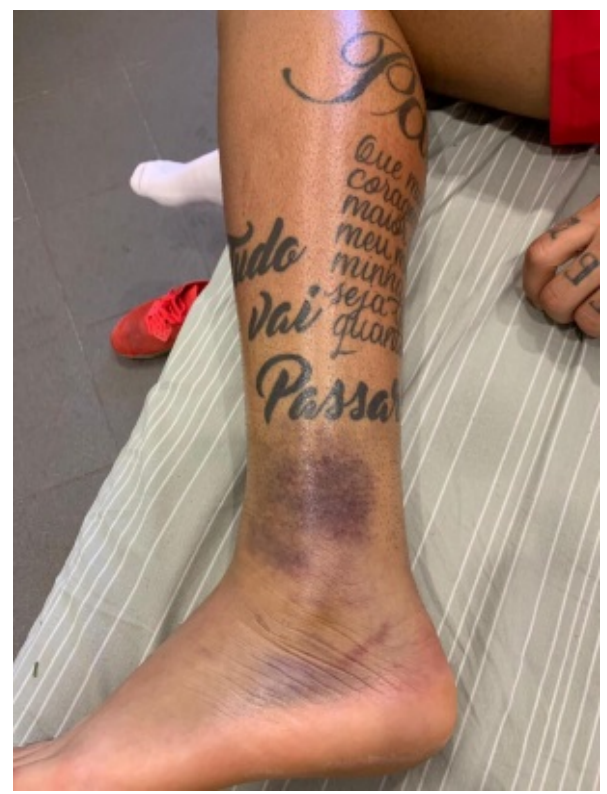

Fig.1. Typical haematoma after rupture of the anterior talofibular and calcaneofibular ligament

A lot of researches have been made regarding the activation of the muscle stabilization, after the feeling of excessive stretching of ligational structures. Most of them find that muscle contraction is too slow to counteract inversion forces. They ware conducted in the range of 18 to $35^{\circ}$ of supination, which is far from the degrees of damage to the connective tissue. P. Vaes and co-authors find that at $50^{\circ}$ of supination motion in upright position, between 105 and 110 milliseconds are needed to complete the entire movement. The muscle activation rate they calculate is about 80 milliseconds. However, these values do not provide information, whether the activated muscle fibers are sufficient to prevent the ligaments from being damaged. Much of the research is also conducted under static conditions, which is largely different from the actual dynamic conditions in which the trauma occurs.

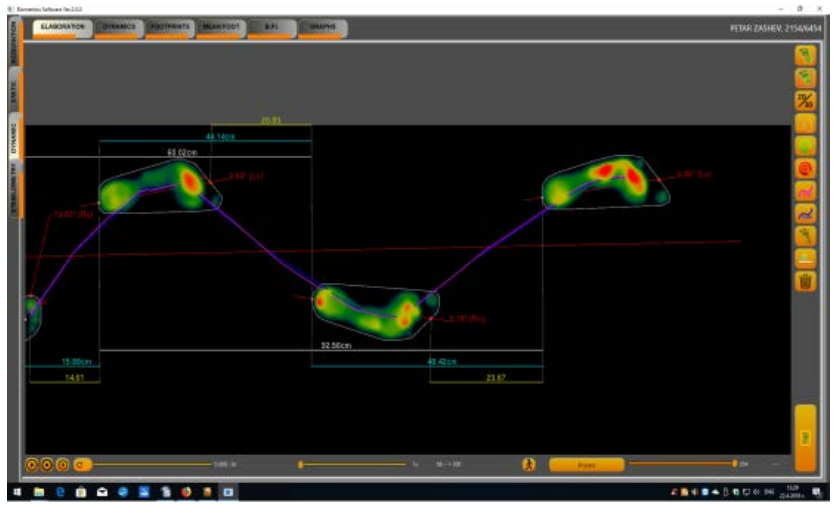

Fig.2. Dynamic evaluation of pacient with distortion of the rightanterior talofibular ligament. 
Proper functional diagnosis and imaging (X-rays, MRI) are necessary to detect the presence of a fracture not only in the area of the ankle joint but also in the middle and front part of the foot. It should be taken into account, that a muscle contracture can mask the joint laxity caused by the rupture of the ligaments. The best method of imaging diagnostics supporting physical examination is MRI.

To determine the condition (both before and after the rehabilitation), baropodometry is used successfully. The system we have (Ultrasensor Walk, Diasu Health Technologies, Rome, Italy) is $200 / 50 \mathrm{~cm}$ in size with 7 sensors each $\mathrm{cm} 2$, and allows to explore both the dynamic (fig. 2) and the satatical load (fig. 3) of the feet, as well as the equilibrium(fig. 6.).

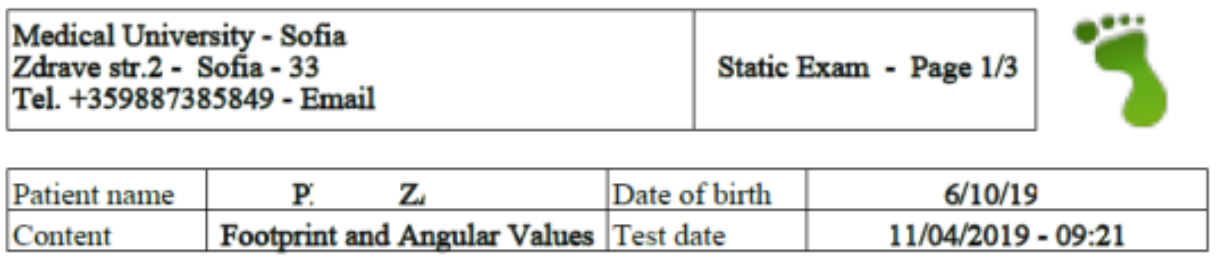

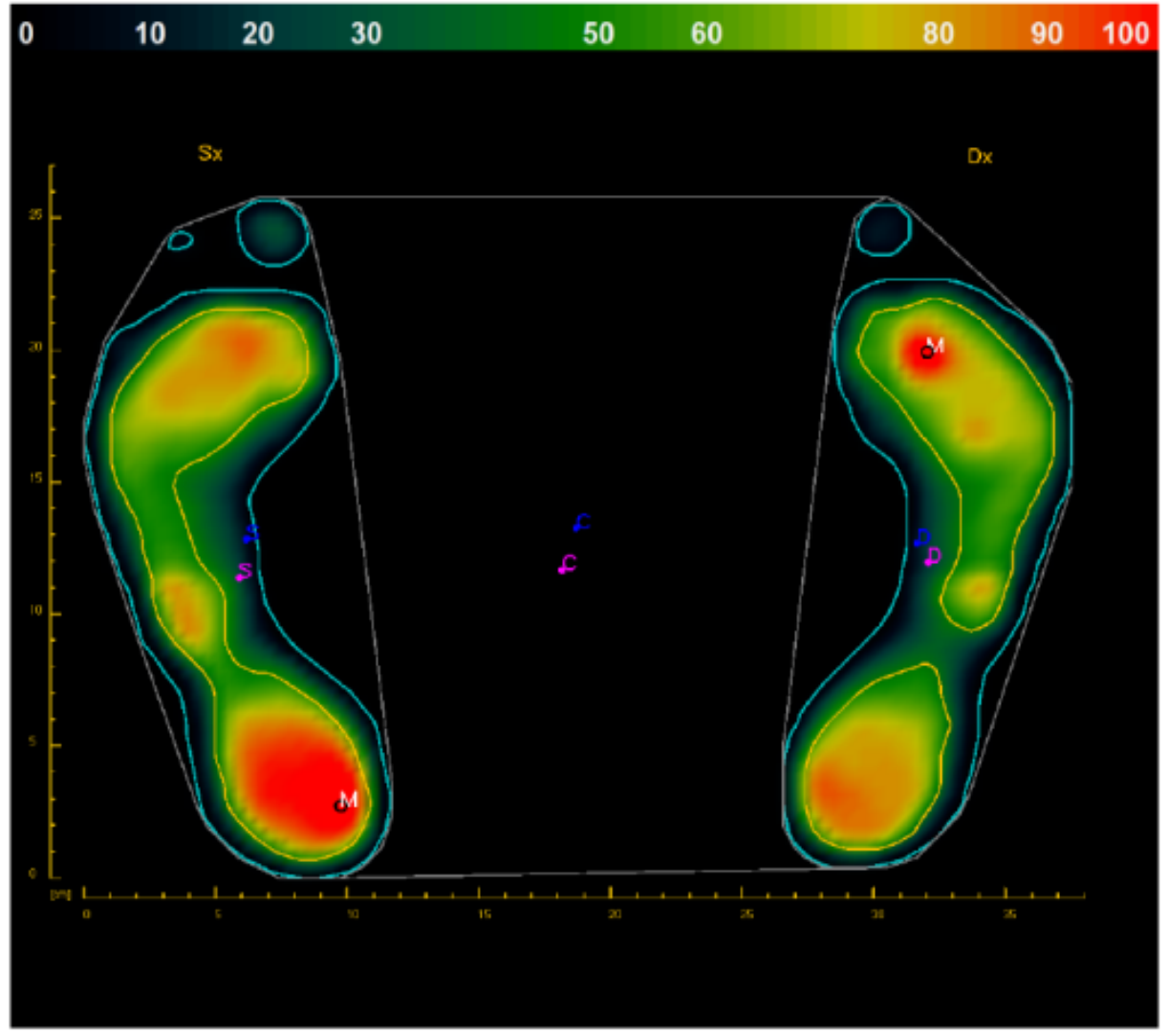

Fig.3. Static evaluation of pacient with distortion of the rightanterior talofibular ligament. 
A large number of authors find that the pressure on the gait analysis is lateralised to the lateral edge of the midfoot and the forefoot, and the patients has a longer total foot contact time. The authors' opinion is that lateral displacement of the loading point at the end-push phase (placing the ankle joint in plantar flexion) is a danger in high-speed movements combined with an effort.

Other authors describe the increased time for the support phase by moving the heel load directly to the middle of the forefoot in patients with chronic ankle instability. They also report a greater load on the front and middle surface of the sole, which is the cause of the maximum loading point laterally.

Third authors note the presence of a gait by turning the legs inwards in patients with chronic instability of the ankle joint.

Medical University - Sofia

Zdrave str.2 - Sofia - 33

Tel. +359887385849 - Email

Static Exam - Page 2/3

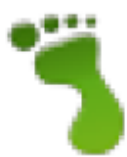

\begin{tabular}{|l|c|l|c|}
\hline Patient name & P $\quad$ Z & Date of birth & 6/10/1 \\
\hline Content & Global and Geometric Data & Test date & 11/04/2019-09:21 \\
\hline
\end{tabular}

\begin{tabular}{|c|c|c|c|c|}
\hline GLOBAL VALUES & Units & $\mathrm{LF}$ & GLOBAL & RG \\
\hline Maximum Pressure & $\mathrm{g} / \mathrm{cm} 2$ & 243.18 & & 239.40 \\
\hline Mean Pressure & $\mathrm{g} / \mathrm{cm} 2$ & 6820 & & 50.56 \\
\hline Foot Load & $\mathrm{kg}$ & 41.66 & & 35.34 \\
\hline Foot Load & $\mathrm{N}$ & 408.69 & & 340.08 \\
\hline Foot Load & $\%$ & 54.10 & & 45.90 \\
\hline Foot Pressor Surface & $\%$ & 54.35 & & 45.65 \\
\hline Foot Pressor Surface & $\mathrm{cm} 2$ & 188.60 & & 158.39 \\
\hline Lenght of Pressor Foot & $\mathrm{cm}$ & 25.97 & & 25.50 \\
\hline Ratio Load/Surface F/R & $\%$ & 0.40 & & 0.46 \\
\hline Ratio Load/Surface R/F & $\%$ & 0.60 & & 0.54 \\
\hline Forefoot Width & $\mathrm{cm}$ & 9.12 & & 8.09 \\
\hline Midfoot Width & $\mathrm{cm}$ & 5.19 & & 7.17 \\
\hline Rearfoot Width & $\mathrm{cm}$ & 7.32 & & 7.05 \\
\hline Disttal Lound & $\%$ & 0.0 & & $\stackrel{0}{n}$ \\
\hline Forefoot Load & $\%$ & 51.50 & & 44.73 \\
\hline Midfoot Load & $\%$ & 3.73 & & 16.52 \\
\hline Rearfoot Load & $\%$ & 44.76 & & 38.75 \\
\hline Divital Precor Surface & $\%$ & 0.00 & & $\operatorname{lng}$ \\
\hline Forefoot Pressor Surface & $\%$ & 45.06 & & 42.39 \\
\hline Midfoot Pressor Surface & $\%$ & 29.13 & & 26.69 \\
\hline Rearfoot Pressor Surface & $\%$ & 25.81 & & 30.92 \\
\hline
\end{tabular}

Fig.4. Static evaluation of pacient with distortion of the rightanterior talofibular ligament. 
Medical University - Sofia

Zdrave str.2 - Sofia - 33

Tel. +359887385849 - Email
Dynamics - Page $2 / 4$

\begin{tabular}{|l|c|l|c|}
\hline Patient name & P Z Z & Date of birth & $6 / 10 / 1 !$ \\
\hline Content & Footprint and Biometrics Data & Test date & $11 / 04 / 2019-09: 26$ \\
\hline
\end{tabular}

\begin{tabular}{|c|c|c|c|c|c|c|}
\hline AVERAGE GLOBAL VALUES & Units & LF & STDDEV & GLOBAL & RG & STDDEV \\
\hline Podalic Area & $\mathrm{cm} 2$ & & & 254.64 & & \\
\hline Max Pressure & $\mathrm{g} / \mathrm{cm} 2$ & $164.36(11)$ & 47.46 & & $141.20(11)$ & 53.97 \\
\hline Mean Pressure & $\mathrm{g} / \mathrm{cm} 2$ & $51.25(11)$ & 8.85 & & $37.92(11)$ & 11.74 \\
\hline Footstoud (o/2) & $\%$ & $\sec 2(11)$ & 30.68 & & $4727(11)$ & 26.71 \\
\hline Foot Load kg & $\mathrm{kg}$ & $40.52(1)$ & 3.66 & & $36.48(11)$ & 3.19 \\
\hline Foot Load N & $\mathrm{N}$ & $397.255(11)$ & 35.91 & & $357.04(11)$ & 31.27 \\
\hline Foot Surface $\mathrm{cm} 2$ & $\mathrm{~cm} 2$ & $118.55(11)$ & 61.09 & & $136.09(11)$ & 59.77 \\
\hline Foot Surface (\%) & $\%$ & $46.55(11)$ & 23.99 & & $53.45(11)$ & 23.47 \\
\hline Lenght of Pressor Foot & $\mathrm{cm}$ & $19.44(11)$ & 8.40 & & $19.98(11)$ & 8.68 \\
\hline Fontmpintmaximum width & $\mathrm{cm}$ & 914.(11) & 1.30 & & $2004(1)$ & 2.00 \\
\hline Rearfoot Load & $\%$ & $28.97(1)$ & 13.04 & & $36.36(11)$ & 6.61 \\
\hline Rearfoutritessor Surface & $\mathrm{cm} 2$ & $36.90(11)$ & 20.43 & & $42 \div 10-(1)$ & 15.83 \\
\hline Rearfoot_Width & $\mathrm{cm}$ & $19.86(11)$ & 41.87 & & $6.67(11)$ & 1.41 \\
\hline Midfoot Load & $\%$ & 27.07 (1) & 11.85 & & $26.93(1)$ & 11.13 \\
\hline Mrumotimssor Surface & $\mathrm{cm} 2$ & 33.64 (11) & 16.92 & & $41.38(11)$ & 16.43 \\
\hline Midfoot Width & $\mathrm{cm}$ & 8.14.(11) & 1.57 & & $8.43(11)$ & 1.88 \\
\hline forefoot Load & $\%$ & $43.95(11)$ & 19.69 & & $36.70(1)$ & 14.17 \\
\hline Foretoor Pressor Surface & $\mathrm{cm} 2$ & $47.95(1)$ & 25.62 & & $52.02(11)$ & 28.21 \\
\hline Forefoot Width & $\mathrm{cm}$ & $7.90(11)$ & 2.36 & & $8.02(11)$ & 2.53 \\
\hline Ratio Load/Surface R/F & $\%$ & $100.83(11)$ & 79.53 & & $117.13(11)$ & 50.89 \\
\hline Ratio Load/Surface F/R & $\%$ & $181.56(11)$ & 208.91 & & $101.83(11)$ & 42.52 \\
\hline
\end{tabular}

Fig.5. Dynamic evaluation of pacient with distortion of the rightanterior talofibular ligament.

In our study, we have received similar results. Fig. 4 and 5 shows the greater load on the healthylimb (which remains even with the dynamic test) and the loadingof the front half of the foot in a static position. During walking, an equalization of the load on the front and the rear of the right foot is observed after a rehabilitation.

The stabilogram (fig. 6.)clearly shows instability in static equilibrium in a Romberg test with parallel legs and closed eyes, executed for $51.2 \mathrm{sec}$. 


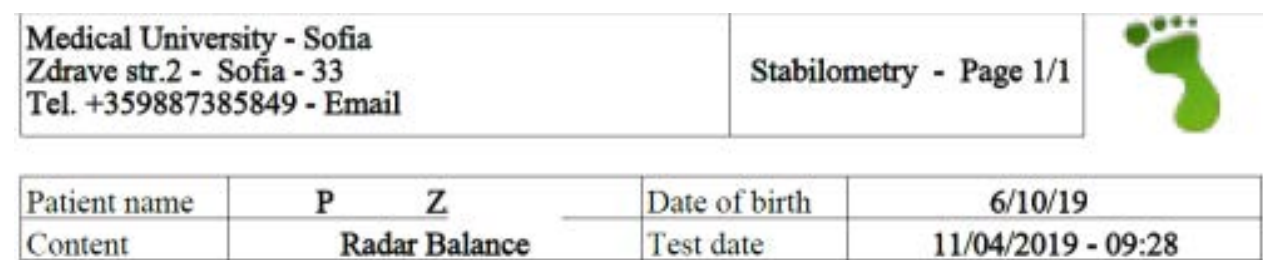

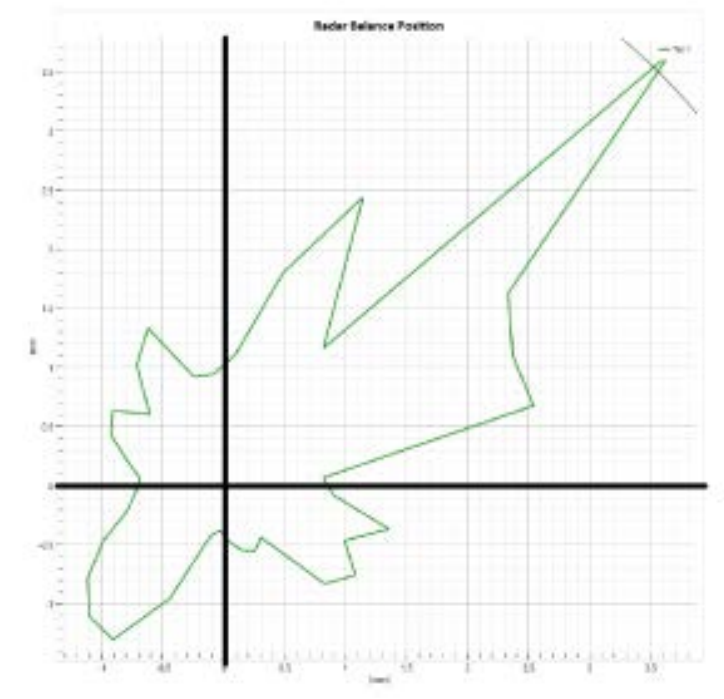

Fig.6. Stabilometry evaluation of pacient with distortion of the rightanterior talofibular ligament.

\section{Physiotherapy treatment}

The ankle sprain algorithm (without fracture, without full tearof the ligaments) is divided into several stages:

- acute period (24 hours after trauma) The PRICE (Protection / Rest / Ice / Compression / Elevation) rule is followed. Immobilization should then be tailored to the start of the recovery process. Our practice shows that the earlier the patient begins the recovery, the more quickly the functional ankle complex will be restored. Early immobilization can be done with splints, orthesis or with a sporting I classic teyping method. Still, the orthopedic cast is practiced, which, in our opinion, is unjustified. Early immobilization, however, has a key role in rapidly restoring the function of damaged ligaments and other tissues in the trauma area!

In the first 48 hours after the trauma, are performed isometricallycontractions in closed kinematics:

- forward and back 2x10, (fig.7)

- forward and right - back and left $1 \times 10$,

- forward and left1x10 back and right $1 \times 10$,

- dynamic reversal in one diagonal $1 \times 10$ and the other diagonal 1x10 (fig.8)

- two laps of the stadium slowly walking (starting from the opposite side of the injured limb). 


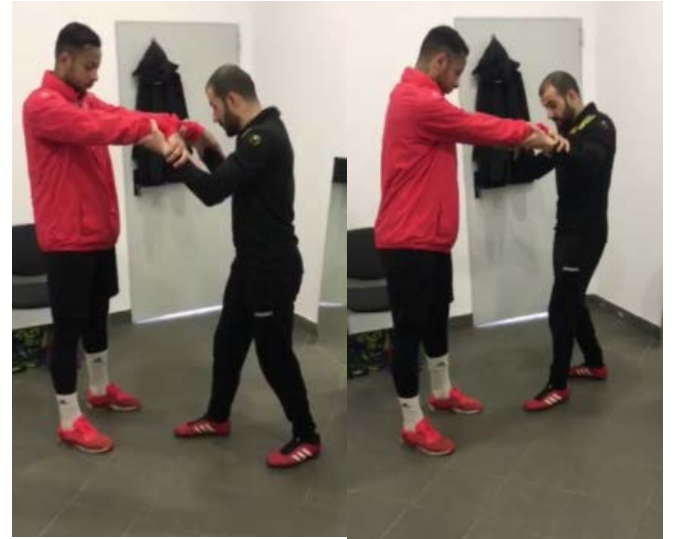

Fig. 7. Pushing the patient front and back.

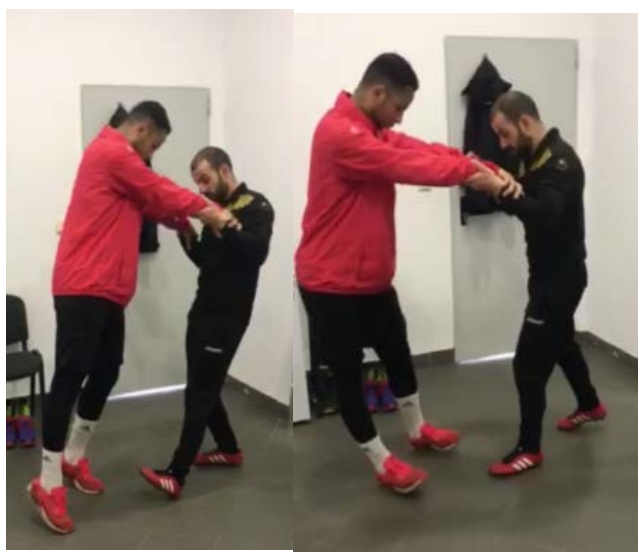

Fig. 8. Dynamic reversal technique.

Day 3.The same thing plus:

- balance of traumatized limb $2 \times 30$ seconds (figure 9).

- two laps from left to right (start again with the right side) (fig.10).

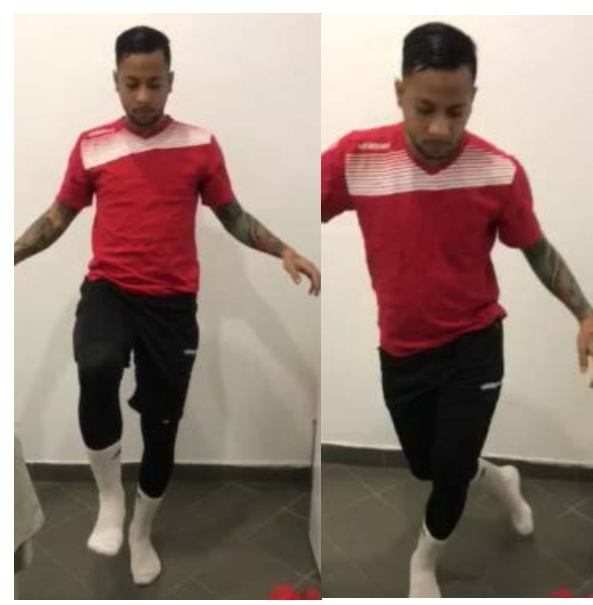

Fig. 9. Balance on the injured leg

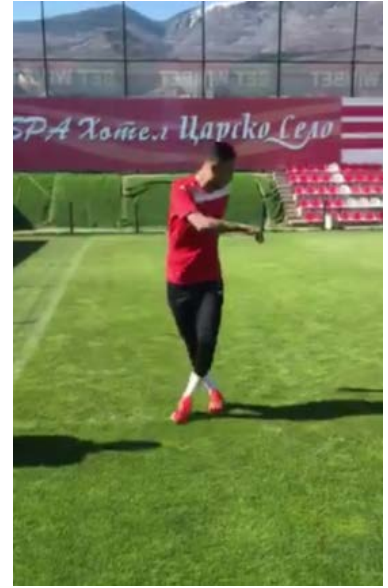

Fig. 10. Sideways cross walking.

Day 4.The same thing, but already with movement, walking is already with a ball, pushing it with a healthy foot has a psycho-emotional influence that the player can easily return to the game.

Day 5. Everything as previous, include:

- PNF models for lower limb without folding of the knee,

- the balance becomes $3 \times 45$ seconds (fig.11),

- walking a lap at a moderate pace, a quick (only on the short sides of the pitch) a total of 4 laps. 


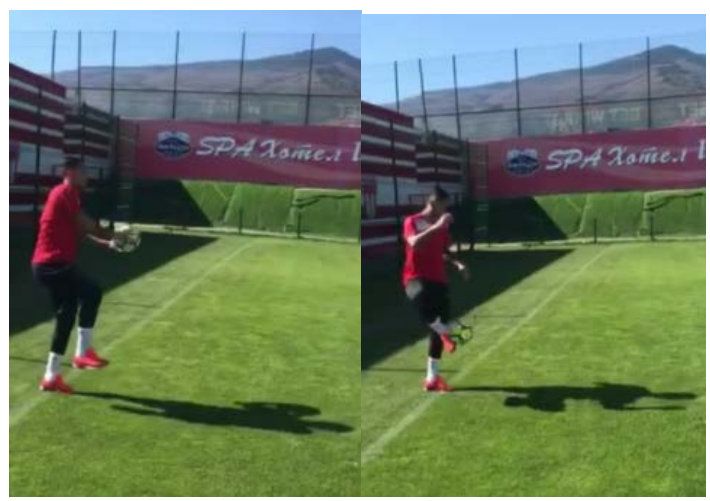

Fig. 11. Balance on the injured legwith ball.

\section{Conclusion}

The ankle joint is part of the complex posture maintenance system. Four types of receptors work on ankle movements: the neuromuscular spindles, the Golgy tendon organs, Ruffini mechanoreceptors and plantar skin mechanoreceptors. The reflexes induced by these receptors are different, depending on whether they use a fast super-segmented unconscious path or a conscious and slower cortical pathway. That is why it is extremely important to restore muscle stabilization - the speed of reaction to maintain the balance, as well as the muscle strength around it.

\section{References}

1. Brockett C. L, G. J Chapman.Biomechanics of the ankle. IN: Orthopaedics and Trauma, Volume 30, Issue 3, June 2016, Pages 232-238

2. Bonnela F., E. Toullecb, C. Mabitc, Y. Tourné. Chronic ankle instability: Biomechanics and pathomechanics of ligaments injury and associated lesions. IN: Orthopaedics \& Traumatology: Surgery \& Research (2010) 96, 424-432

3. Vaes P., W. Duquet, B. Van Gheluwe. Peroneal Reaction Times and Eversion Motor Response in Healthy and Unstable Ankles. IN: Journal of Athletic Training. 2002 Oct-Dec; 37(4): 475-480.

4. Morrison K. E., T. W. Kaminski. Foot Characteristics in Association With Inversion Ankle Injury. IN: Journal of Athletic Training.2007 Jan-Mar; 42(1): 135-142.

\section{Corresponding author:}

1. Victor Valchanov - physiotherapists, Football Club "Tsarsko selo", Sofia, Bulgaria

2. Assoc. prof. Stefan Yanev, PT, PhD, Department of Kinesitherapy, Faculty of Public Health, Medical University - Sofia E-mail: s.janev@foz.mu-sofia.bg 